\title{
Laboratory investigations in lipidology
}

\author{
J A Rusch, BSc, MB BCh; C L Hudson, MB ChB, FCPath Chem (SA); A D Marais, MB ChB, FCP (SA) \\ Division of Chemical Pathology, Department of Pathology, Faculty of Health Sciences, University of Cape Town and \\ National Health Laboratory Service, Groote Schuur Hospital, Cape Town, South Africa
}

Corresponding author: A D Marais (david.marais@uct.ac.za)

\begin{abstract}
Advances in the causes of disorders of lipid metabolism, and effective intervention in atherosclerosis with medication, have increased the reliance on laboratory investigation in clinical practice. The conventional lipid profile, comprising fasting triglyceride, total cholesterol, high-density lipoprotein (HDL) cholesterol and low-density lipoprotein (LDL) cholesterol, suffices for screening persons at risk of atherosclerosis. Additionally, lipoprotein (a) measurement enhances risk assessment and could explain atherosclerosis with a desirable lipid profile. Certain rare disorders in sterol and fatty acid metabolism do not alter the conventional lipid profile. Non-fasting samples are gaining popularity, as the triglycerides, although mildly increased, signify atherogenic lipoprotein remnant accumulation. Apoprotein A1 (apo A1) and apo B concentrations parallel HDL cholesterol and LDL cholesterol, respectively, and are of value in disorders of these lipoproteins. In severe dyslipoproteinaemia, special investigations establish the cause of the disease and may allow selection of better treatment. In such cases, not only apoprotein concentrations, but also enzyme or cell function, as well as genetic investigations, are relevant. The most important genetic disorders to recognise are familial hypercholesterolaemia, dysbetalipoproteinaemia and chylomicronaemia. Expertise in lipidology is limited in South Africa, but specialist centres can provide clinical and laboratory support to ensure best management of severe disorders.
\end{abstract}

S Afr Med J 2018;108(4):266-270. DOI:10.7196/SAMJ.2018.v108i4.13233

Although laboratory investigation is essential for diagnosis and management, it also introduces advances in science, thereby enhancing patient care. Investigations should not, however, strain limited resources. Modest changes in the lipid profile impact on atherosclerosis risk, and undesirable values, are highly prevalent in both sexes worldwide. ${ }^{[1]}$ More extreme dyslipidaemias identify individuals at higher risk of atherosclerosis. Lipid disorders, including pancreatitis and metabolic disorders, should also be considered in other clinical settings. The purpose of this article is to provide the medical practitioner with an understanding of laboratory investigations in lipidology.

\section{Lipids}

Lipids are water-insoluble compounds including sterols (cholesterol) and fatty acids (FAs) (typically esterified as triglycerides and phospholipids). Lipids serve many functions in the cell: membrane formation, steroid hormone and bile acid production, and the provision and storage of energy. Lipids are transported as lipoproteins, but albumin transports non-esterified FAs.

\section{Fatty acids}

FAs are carboxylic acids comprising mostly saturated but also mono- or polyunsaturated carbon to carbon bonds. Short-chain $(\leq 6 \mathrm{C})$ and medium-chain $(8-12 \mathrm{C})$ FAs are generally metabolised intracellularly. Long-chain (14 - 22 C) FAs are found in triglycerides and phospholipids. The latter influences inflammation and thrombosis through prostaglandins. Essential FAs (linoleic acid and alpha-linolenic acid) are sourced from plants. Plants also contain very-long-chain FAs and branch-chain FAs, which are metabolised in peroxisomes. Dietary FAs, as well as those generated in the gut microbiota, significantly influence health. ${ }^{[2]}$

\section{Triglycerides}

Triglycerides consist of three long-chain FAs esterified to glycerol.
In animals they form fats. In plants they form oils, as unsaturated bonds confer liquid properties. Triglycerides are also synthesised in hepatocytes and adipocytes. During fasting, free FAs mobilise from adipocytes to the liver, which releases triglycerides and ketones as sources of energy.

\section{Phospholipids}

Phospholipids resemble triglycerides, but a terminal FA is substituted by a phosphate and choline, ethanolamine, serine or inositol. Phospholipids are the main structural component of cell membranes, coat lipoproteins and emulsify dietary fat.

\section{Sterols}

Cholesterol is vital to animal cell membranes, functions in signalling during embryogenesis, and is the substrate for steroid hormone and bile acid (BA) synthesis. Cholesterol is absorbed from the diet or synthesised de novo by cells. Beta-hydroxy beta-methylglutaryl (HMG)-CoA reductase, the rate-limiting enzyme in biosynthesis, is upregulated by cholesterol depletion in cells and inhibited by statins. Oxysterols regulate sterol and other metabolic pathways, and may be found in atherosclerotic plaques. Precursor sterols and plant sterols (beta-sitosterol and campesterol), BAs and an intermediate product, cholestanol, occur in trace quantities in plasma. BAs, via signalling pathways, may also regulate glucose and lipid metabolism, and energy homeostasis. The enterohepatic circulation of BAs is disrupted in liver dysfunction, resulting in hypercholanaemia.

\section{Lipoproteins}

Lipoproteins are spherical particles comprising a core of triglycerides and cholesterol esters (CEs), and a surface of phospholipid and unesterified cholesterol (UC) in which apoproteins reside. Apoproteins facilitate lipoprotein assembly, modulation and clearance. Lipoproteins are classified by ultracentrifugation into 
five major types, with increasing density and decreasing size: chylomicrons (CMs), very low-density lipoprotein (VLDL), intermediate-density lipoprotein (IDL), low-density lipoprotein (LDL) and high-density lipoprotein (HDL). The density of the particle depends on the lipid (less dense) and protein (denser) content. During fasting, VLDL carries the majority of triglyceride and LDL carries the majority of cholesterol. Circulating CMs after a meal raise triglyceride levels. In the circulation, lipoproteins undergo continuous modification in a flux through interconnected pathways (Fig. 1).

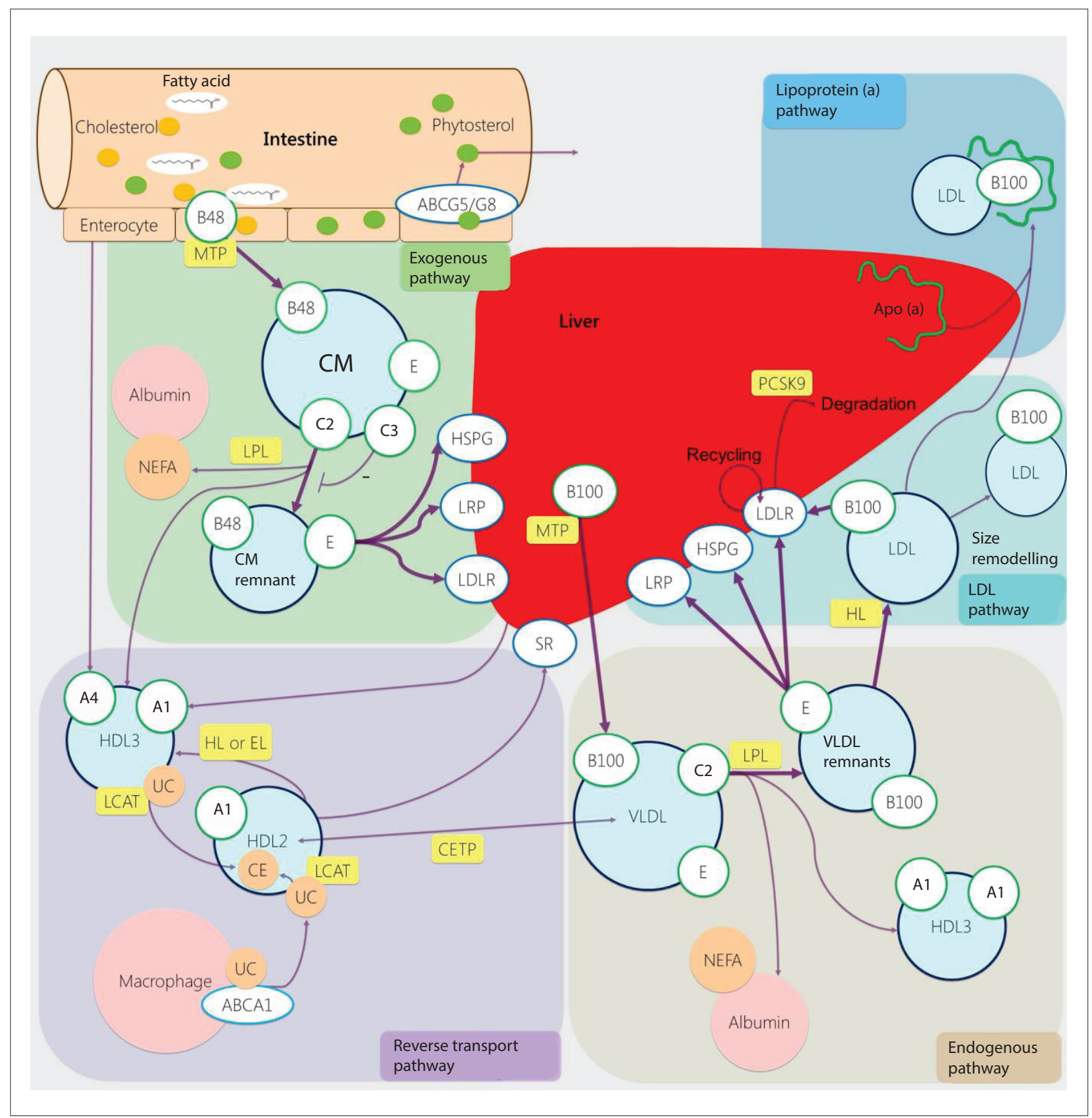

Fig. 1. Lipoprotein metabolic pathways. Exogeneous pathway: after the intestinal absorption and assembly on apo B48 of dietary fatty acids and sterols, chylomicrons transport lipids to tissues and finally the liver. Endogeneous pathway: the liver assembles triglycerides and cholesterol on apo B100 in very low-density lipoprotein (VLDL) during fasting, with recycling of remnants. Low-density lipoprotein (LDL) pathway: the endogeneous pathway ends with the supply of cholesterol to tissues on demand through LDL-receptor expression. Reverse cholesterol transport pathway: moves cholesterol from cells to the liver either directly (scavenger receptor) or indirectly (VLDL) by cholesterol ester transfer protein. Apo A4 synthesised in enterocytes, as well as other apoproteins, assimilates in high-density lipoprotein. Lipoprotein (a) pathway: highly variable in humans. Albumin pathway: non-esterified fatty acid complex with albumin in plasma. (A1, A4, B48, B100, E = apoproteins; ABCA1 and ABCG5/G8 = ATP-binding casette transporter proteins; $C E=$ cholesterol ester; $C E T P=$ cholesterol ester transfer protein $C M=$ chylomicron; $F A=$ fatty acid; $H S P G=$ heparan sulphate proteoglycans; $H D L=$ high-density lipoprotein; $I D L=$ intermediate-density lipoprotein $L C A T=$ lecithin:cholesterol acyl transferase; $L P L=$ lipoprotein lipase; $L D L=$ low-density lipoprotein; $L D L R=L D L$ receptor; $L D L R P=L D L$ receptor-like protein; $N E F A=$ non-esterified fatty acid; $S R=$ scavenger receptor $B 1 ; U C=$ unesterified cholesterol; VLDL $=$ very low-density lipoprotein.) 
In the exogenous pathway, lipoprotein lipase (LPL), held on the vascular endothelium by heparan sulphate proteoglycans (HSPGs), hydrolyses triglycerides (in the presence of apoprotein C2 (apo C2)), releasing FAs and CM-remnants in the process. Excessive phospholipid on shrinking CMs can depart with apo A1, forming HDL.

In the endogenous pathway, hepatocytes assemble triglycerides onto apo B100, forming VLDL. Similar to CMs, circulating VLDL undergoes lipolysis, resulting in remnants, while generating some HDL. These remnants, IDL, are cleared by the liver through apo E binding HSPG, LDL receptor-like protein (LRP) and the LDL receptor (LDLR). Some remnants are processed to LDL, on which apo B100, now the sole apoprotein, is in the correct conformation to bind the LDLR (expressed by cells that require cholesterol).

HDL transports cholesterol in the reverse pathway, predominantly with apo A1, which assimilates phospholipid, but also apo A4 and apo E. Cells respond to apo A1 by releasing cholesterol and phospholipid through adenosine-binding cassette transporter protein (ABCA1). Lecithin:cholesterol acyl transferase (LCAT) enriches HDL with cholesterol by forming CE that moves to its core. CEs may be exchanged by cholesterol ester transfer protein (CETP) to triglyceride-rich lipoproteins, destined for the liver. HDL can also deliver CEs directly to the liver via scavenger receptor B1 (SR).

Lipoprotein (a) (Lp (a)) resembles LDL but contains covalently bound apo (a). Being homologous to plasmin, it may impair thrombolysis, explaining its modest independent impact on atherosclerosis.

\section{Laboratory investigations}

Most lipidological investigation assesses cardiovascular disease (CVD) risk and monitors treatment response. Atherosclerosis is associated with increasing LDL and remnants and decreasing HDL concentrations. Hypertriglyceridaemia predicts pancreatitis. Less commonly, genetic disorders with severe dyslipidaemias may occur. Rarely, FAs and sterol disorders may be present without disturbing the conventional lipid profile.

\section{Sampling}

Approximately $3 \mathrm{~mL}$ of blood for routine analysis is collected in a plain tube (yellow- or red-capped) to analyse the serum lipids. CMs and VLDL scatter light and result in turbidity (triglyceride $>2.5 \mathrm{mmol} / \mathrm{L}$ ). Frank lipaemia indicates severe hypertriglyceridaemia (triglyceride $>10 \mathrm{mmol} / \mathrm{L}$ ). In effusions, cholesterol may differentiate transudates from exudates; triglyceride may indicate chylothorax. ${ }^{[3,4]}$

\section{Routine investigations}

Secondary causes for dyslipidaemias should be excluded or treated before full lipidological work-up (Table 1). Hypercholesterolaemia may be due to nephrotic syndrome or hypothyroidism. Hypertriglyceridaemia may be caused by alcohol or diabetes.

The conventional profile comprises triglyceride, total cholesterol (TC) and HDL cholesterol, which are measured by enzymatic colorimetric assays, and LDL cholesterol, which can be calculated in fasting samples or measured directly. Cholesterol esterase ensures that cholesterol oxidase reacts with all cholesterol. In homogenous HDL cholesterol assays, dextran sulphate selectively forms watersoluble complexes with LDL, VLDL and CMs, which resist modified enzymes.

Clinically insignificant differences are found in the TC and HDL cholesterol results of fasting or non-fasting individuals. Triglycerides are traditionally measured in the fasting state because the Friedewald equation (Box 1) will approximate the LDL cholesterol
Box 1. Friedewald equation to calculate LDLC $(\mathrm{mmol} / \mathrm{L})$ LDLC = TC - HDLC $-(\mathrm{TG} / 2.2)$, where (TG/2.2) is an estimation of VLDLC

LDLC = low-density lipoprotein cholesterol; TC = total cholesterol; HDLC $=$ high density lipoprotein cholesterol; TG = triglyceride; $\mathrm{VLDL}=$ very low-density lipoprotein cholesterol.

when triglyceride values are $<4.5 \mathrm{mmol} / \mathrm{L}$, provided that remnants, Lp (a) and Lp X are absent. LDL cholesterol can be measured directly by homogenous assays or ultracentrifugation.

TC results can vary in an individual by as much as $14 \%$ owing to factors such as stress, illness, posture, seasonal variation and interlab variability. ${ }^{[5]}$ More than one TC measurement is advised when decisions are to be made in moderate dyslipidaemias. Even greater variability may be demonstrated by HDL cholesterol measurement. ${ }^{[6]}$

\section{Extended lipid profile}

Apo A1, apo B and Lp (a) are measured immunoturbidimetrically. Apo A1 is the major protein of HDL, whereas apo B is the major protein of LDL, accounting for $95 \%$ of plasma apo B. Apo B measurements are a surrogate for lipoprotein particle concentration (number). For the same LDL concentration, small LDL will have a larger particle number. ${ }^{[7]}$ Strong associations between CVD and LDL particle number, or high concentrations of $\mathrm{Lp}$ (a), provide a rationale for advanced testing in CVD management.

\section{Special investigations \\ Electrophoresis}

Routine ultracentrifugation is impracticable, but electrophoresis can demonstrate lipoprotein fractions in small sample volumes and remains useful for the diagnosis of several dyslipidaemias. The traditional agarose gels can diagnose Fredrickson type 1 (chylomicronaemia), type 3 (dysbetalipoproteinaemia), Lp X and sulphated cholesterol in X-linked ichthyosis. Lp X is an abnormal lipoprotein that is formed in cholestasis or LCAT deficiency. Deficiencies or absences of lipoproteins are also readily demonstrated. This includes abetalipoproteinaemia, hypobetalipoproteinaemia and Tangier disease (analphalipoproteinaemia).

Non-denaturing polyacrylamide gel electrophoresis is less well established but discriminates LDL sizes and detects dysbetalipoproteinaemia well..$^{[8]}$ Small and dense LDL is associated with insulin resistance and diabetes, is more susceptible to oxidation and enters arterial walls more readily. CVD risk is increased twoto three-fold. ${ }^{[7,9,10]}$ Nuclear magnetic resonance spectroscopy, density gradient ultracentrifugation and ion mobility analysis also characterise LDL further.

\section{Genetic testing}

Genotyping should be used judiciously and only where it impacts management. This includes preconception counselling and identifying carriers of severe recessive disorders. Genetic diagnosis, along with functional fibroblast studies, is warranted if homozygous familial hypercholesterolaemia (FH) is considered. With decreasing costs of sequencing and the future possibility of genome editing, genotyping may become routine.

\section{Sterols}

Unlike cholesterol, present in millimolar concentration, its precursors, products and plant analogues are only found at micromolar concentration in plasma. Non-cholesterol sterols are typically detected by gas chromatography-mass spectrometry (GC-MS) or GC-flame ionisation detection (GC-FID). Cholesterol biosynthetic 
Table 1. Dyslipidaemias and dyslipoproteinaemias with their primary and secondary causes

\begin{tabular}{|c|c|c|c|}
\hline Lipid $(\mathrm{mmol} / \mathrm{L})$ & Lipoprotein (lipid) involved & Secondary causes & Primary causes \\
\hline TG $1.7-2.5$ & VLDL & $\begin{array}{l}\text { Dysglycaemia } \\
\text { Alcohol } \\
\text { Obesity } \\
\text { Corticosteroids } \\
\text { Retinoids }\end{array}$ & Monogenic variants (e.g. LPL deficiency) \\
\hline TG $2.5-5.0$ & $\mathrm{VLDL} \pm \mathrm{IDL}$ & $\begin{array}{l}\text { Dysglycaemia } \\
\text { Alcohol } \\
\text { Obesity } \\
\text { Corticosteroids } \\
\text { Retinoids }\end{array}$ & $\begin{array}{l}\text { Polygenic variants (mild) } \\
\text { Monogenic variants } \\
\text { (e.g. LPL deficiency) } \\
\text { Dysbetalipoproteinaemia }\end{array}$ \\
\hline $\begin{array}{l}\text { TG } 5.0 \text { - } 15.0 \text { (often with } \\
\text { hypercholesterolaemia) }\end{array}$ & VLDL, IDL \pm CM & $\begin{array}{l}\text { Dysglycaemia } \\
\text { Alcohol } \\
\text { Obesity } \\
\text { Corticosteroids } \\
\text { Retinoids }+ \\
\text { genetic predisposition }\end{array}$ & $\begin{array}{l}\text { Polygenic variants (mild) } \\
\text { Monogenic variants } \\
\text { (e.g. LPL deficiency) } \\
\text { Dysbetalipoproteinaemia }\end{array}$ \\
\hline $\begin{array}{l}\text { TG }>15.0 \text { (usually with } \\
\text { hypercholesterolaemia) }\end{array}$ & CM, VLDL & $\begin{array}{l}\text { Genetic predisposition + } \\
\text { metabolic stress }\end{array}$ & $\begin{array}{l}\text { Polygenic variants (mild) } \\
\text { Monogenic variants } \\
\text { (e.g. LPL deficiency), more commonly } \\
\text { monogenic } \\
\text { (LPL, apo C2 apo Av, LMF-1, } \\
\text { GPI-HBP-1) } \\
\text { Dysbetalipoproteinaemia }\end{array}$ \\
\hline $\mathrm{TC}<2.0$ & LDL, VLDL, CM & $\begin{array}{l}\text { Acute-phase response } \\
\text { Malnutrition } \\
\text { Malabsorption }\end{array}$ & $\begin{array}{l}\text { Apo B truncations } \\
\text { MTTP deficiency }\end{array}$ \\
\hline $\begin{array}{l}\text { TC } 2.0 \text { - } 5.0 \text { (atherosclerosis } \\
\text { setting) }\end{array}$ & $\begin{array}{l}\mathrm{HDL}(<0.7 \mathrm{mmol} / \mathrm{L}) \\
\mathrm{Lp}(\mathrm{a})\end{array}$ & Acute-phase response & $\begin{array}{l}\text { ABCA1, LCAT, apo A1 } \\
\text { Genetic variants }\end{array}$ \\
\hline $\begin{array}{l}\text { TC } 2.0 \text { - } 5.0 \text { (other clinical } \\
\text { presentations) }\end{array}$ & $\begin{array}{l}\text { Unusual lipids } \\
\text { VLCFA } \\
\text { BCFA } \\
\text { Cholesterol sulphate } \\
\text { Cholestanol }\end{array}$ & Not applicable & $\begin{array}{l}\text { Adrenoleukodystrophy } \\
\text { Refsum disease } \\
\text { X-linked ichthyosis } \\
\text { CTX }\end{array}$ \\
\hline TC $5.0-7.5$ & LDL (rarely HDL elevates TC) & $\begin{array}{l}\text { Diet } \\
\text { Hypothyroidism } \\
\text { Nephrotic syndrome } \\
\text { Lp X }\end{array}$ & $\begin{array}{l}\text { Polygenic } \\
\text { Hyperalphalipoproteinaemia: CETP, apo C3, EL } \\
\text { and SR-B1 deficiencies }\end{array}$ \\
\hline TC $7.5-15.0$ & LDL & $\begin{array}{l}\text { Lp X } \\
\text { Nephrotic syndrome } \\
\text { Hypothyroidism }\end{array}$ & $\begin{array}{l}\text { FH: LDL }>5.0 \mathrm{mmol} / \mathrm{L} \\
\text { LDL-R, FDB and ARH, PCSK9 gain of function } \\
\text { mutations } \\
\text { Phytosterolaemia }\end{array}$ \\
\hline $\mathrm{TC}>15.0$ & LDL & Lp X & $\begin{array}{l}\text { Homozygous FH, ARH } \\
\text { Phytosterolaemia }\end{array}$ \\
\hline \multicolumn{4}{|c|}{ 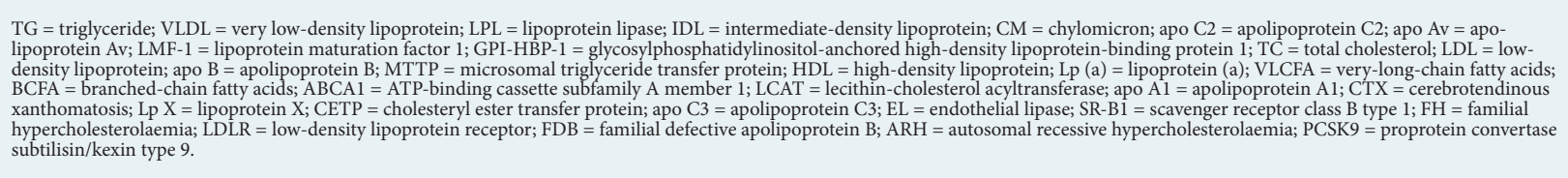 } \\
\hline
\end{tabular}

errors elevating levels of 7-dehydrocholesterol, lathosterol or desmosterol, should be considered in the setting of congenital malformations and impaired development. A spectrophotometric assay for 7-dehydrocholesterol is less expensive to diagnose SmithLemli-Opitz syndrome. ${ }^{[11]}$

Markedly elevated levels of sitosterol and campesterol confirm phytosterolaemia, an autosomal recessive disorder mimicking $\mathrm{FH}$. The adenosine triphosphate (ATP)-binding cassette sub-family G5 or G8 mutations prevent the excretion of plant sterols.
Cerebrotendinous xanthomatosis is an autosomal recessive disease caused by a deficiency of sterol 27-hydroxylase. Impaired BA synthesis is accompanied by increased formation of cholestanol. There may be massive tendon xanthomata, leukodystrophy and cataracts without significant dyslipidaemia. BA supplements can be of benefit because they suppress BA synthesis.

\section{Fatty acids}

Determining the profile of physiologically and nutritionally 
important FAs is of limited use in clinical practice. However, certain rare peroxisomal disorders are diagnosed by identifying abnormal FAs. Very-long-chain FAs impair adrenal and neuronal function in $\mathrm{X}$-linked adrenoleukodystrophy. In Refsum disease, branched-chain FAs accumulate, resulting in neurological, cutaneous and ophthalmic manifestations.

\section{Enzyme analysis}

Enzyme analysis is rarely required but can shed light on disease mechanismsandimprovemanagement.LPLdeficiencyisdemonstrated in plasma by displacement of LPL from heparan sulphate proteoglycans on endothelial cells after heparin injection. The autosomal recessive disorder, characterised by marked hyperchylomicronaemia and a corresponding hypertriglyceridaemia, can be due to errors in LPL, its cofactors apo C2 and apo A5, or its transporters lipase maturation factor 1 and glycosylphosphatidylinositol-anchored highdensity lipoprotein-binding protein 1 (GPI-HBP-1). If apo C2 is deficient, supplementation by fresh-frozen plasma can alleviate severe hypertriglyceridaemia and pancreatitis.

Other enzyme studies of interest include LCAT activity in the setting of low HDL cholesterol, and lysosomal acid lipase in Wolman disease and CE storage disease. The latter presents as a mixed hyperlipidaemia with severe liver enzyme derangement, and responds to recombinant lysosomal acid lipase.

\section{Cell culture}

Fibroblast cultures are performed at few centres but may be crucial for diagnosis. The neurovisceral storage disorder, Niemann-Pick type $\mathrm{C}$, sequesters cholesterol and glycosphingolipid in lysosomes. Filipin demonstrates the unesterified cholesterol, which cannot be processed, by fluorescence. ${ }^{[12]}$ LDL uptake studies in fibroblasts can reflect LDL receptor function in homozygous $\mathrm{FH}$ and can predict the response to proprotein convertase subtilisin/kexin type 9 (PCSK9) neutralising monoclonal antibodies.

\section{Discussion}

This review addresses dyslipidaemia broadly to ensure recognition of a wide range of disorders. The majority of clinicians, however, will mainly manage persons at risk for atherosclerosis and pancreatitis. Clinical settings, in which specific lipid disorders should be recognised, are also briefly mentioned. The dyslipidaemias and dyslipoproteinaemias and their causes are summarised in Table 1.

\section{Guidelines}

Guidelines allow for reasonable practice based on CVD risk when serving the general population, but do not necessarily adequately advise on the management of severe genetic disorders. Every effort must be made to reach a diagnosis in the case of these disorders for best management decisions. This may involve referral to specialist centres.

\section{Cost-effective testing}

A full lipogram (TC, HDL cholesterol, LDL cholesterol and triglycerides), costing $\sim$ ZAR300, is recommended for the initial diagnosis of dyslipidaemia. ${ }^{[13]}$ LDL cholesterol (ZAR110) or TC (ZAR80) alone can be used in the follow-up of patients with pure hypercholesterolaemia. A full lipogram is advocated for follow-up where increased LDL cholesterol is not the only abnormality in the lipid profile.

Despite the proven use of these established tests in CVD risk prediction and management, the burden of CVD events remains high. Additional lipidologic (apo B) and inflammatory markers
(C-reactive protein) may aid in addressing borderline or residual risk. Guidelines differ in their application. Apo B measurement (ZAR130) estimates LDL particle number. Whether it is superior to non-HDL cholesterol or total/HDL cholesterol ratio for CVD risk prediction is controversial. More data are clearly needed on the use of advanced lipoprotein tests, as well as non-HDL cholesterol and total/HDL cholesterol ratio as targets of therapy for common dyslipidaemias. ${ }^{[13]}$

\section{Referral criteria}

Severe dyslipidaemias, unexplained cutaneous or tendon xanthomata, problematic management, premature atherosclerosis and suspected metabolic errors all constitute reasons for referral. Heterozygous FH should be considered if TC concentration is $>7.5 \mathrm{mmol} / \mathrm{L}$. Most patients respond to statin therapy, but poorly controlled patients may require additional treatment following specialist work-up.

Extreme dyslipidaemias for referral and special investigation include: TC $>15 \mathrm{mmol} / \mathrm{L}$, LDL cholesterol $>12 \mathrm{mmol} / \mathrm{L}$ and triglyceride $>10 \mathrm{mmol} / \mathrm{L}$, unusually low TC $(<2.0 \mathrm{mmol} / \mathrm{L})$, LDL cholesterol $(<1.5 \mathrm{mmol} / \mathrm{L})$ or HDL cholesterol $(<0.7 \mathrm{mmol} / \mathrm{L}) .^{[14]}$

For severe atherogenic dyslipidaemias, or those causing pancreatitis, effective new treatment strategies have been developed. Specialist centres, where clinical and laboratory expertise converge, are important for recognition and accurate diagnosis. Increasing consideration of severe or unusual lipid disorders will drive a relevant diagnostic repertoire, clinical expertise and patient care for South Africa.

\section{Acknowledgements. None.}

Author contributions. All authors confirm that they have contributed to the intellectual content of this article and have met the following three requirements: (i) significant contributions to the conception and design, acquisition of data, or analysis and interpretation of data; (ii) drafting or revising the article for intellectual content; and (iii) final approval of the published article.

Funding. None.

Conflicts of interest. None.

1. World Health Organization. Global health observatory data. http://www.who.int/gho/ncd/risk factors/cholesterol_mean_text/en/(accessed 3 January 2018).

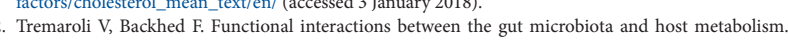
Tremaroli V, Backhed F. Functional interactions between the gut mi
Nature 2012;489(7415):242-249. https://doi.org/10.1038/nature11552

3. McGrath EE, Blades Z, Anderson PB. Chylothorax: Aetiology, diagnosis and therapeutic options. Respir Med 2010;104(1):1-8. https://doi.org/10.1016/j.rmed.2009.08.010

4. Heffner JE, Sahn SA, Brown LK. Multilevel likelihood ratios for identifying exudative pleural effusions. Chest 2002;121(6):1916-1920. https://doi.org/10.1378/chest.121.6.1916

5. Cooper GR, Myers GL, Smith SJ, Schlant RC. Blood lipid measurements. Variations and practical utility. JAMA 1992;267(12):1652-1660. https://doi.org/10.1016/0020-7292(92)90669-a

6. Bachorik PS, Cloey TA, Finney CA, Lowry DR, Becker DM. Lipoprotein-cholesterol analysis during screening: Accuracy and reliability. Ann Intern Med 1991;114(9):741-747. https://doi. org/10.7326/0003-4819-114-9-741

7. Lamarche B, Tchernof A, Moorjani S, et al. Small, dense low-density lipoprotein particles as a predictor of the risk of ischemic heart disease in men. Prospective results from the Quebec Cardiovascular Study. Circulation 1997;95(1):69-75. https://doi.org/10.1161/01.cir.95.1.69

8. Blom DJ, Byrnes $\mathrm{P}$, Jones $\mathrm{S}$, Marais $\mathrm{AD}$. Non-denaturing polyacrylamide gradient gel electrophoresis for the diagnosis of dysbetalipoproteinemia. J Lipid Res 2003;44(1):212-217. https://doi.org/10.1194 jlr.d200013-jlr200

9. Koba S, Hirano T. Small dense low-density lipoprotein in Japanese men with coronary artery disease. Ann Intern Med 2000;132(9):762. https://doi.org/10.7326/0003-4819-132-9-200005020-00024

10. St-Pierre AC, Ruel IL, Cantin B, et al. Comparison of various electrophoretic characteristics of LDL particles and their relationship to the risk of ischemic heart disease. Circulation 2001;104(19):22952299. https://doi.org/10.1161/hc4401.098490

11. Solomon GAE, Jones G, de Jong G, Marais AD. Biochemical and genetic diagnosis of Smith-LemliOpitz syndrome in South Africa. S Afr J Child Health 2015;9(1):23-26. https://doi.org/10.7196/ sajch.771

2. Bornig H, Geyer G. Staining of cholesterol with the fluorescent antibiotic 'filipin'. Acta Histochem 1974;50(1):110-115.

13. Mora S. Advanced lipoprotein testing and subfractionation are not (yet) ready for routine clinical use. Circulation 2009;119(17):2396-2404. https://doi.org/10.1161/circulationaha.108.819359

14. Klug EQ, Raal FJ, Marais AD, et al. South African dyslipidaemia guideline consensus statement. S Afr Fam Pract 2015;57(2):22-31. https://doi.org/10.1080/20786204.2013.10874296

Accepted 5 March 2018. 